\title{
Pulmonary hypertension in patients with pulmonary fibrosis awaiting lung transplant
}

\author{
A.F. Shorr*, J.L. Wainright ${ }^{\#}$, C.S. Cors ${ }^{\#}$, C.J. Lettieri" and S.D. Nathan ${ }^{+}$
}

ABSTRACT: Pulmonary hypertension (PH) may complicate idiopathic pulmonary fibrosis (IPF) but the prevalence of PH in IPF remains undefined. The present authors sought to describe the prevalence of PH in IPF.

The lung transplant registry for the USA (January 1995 to June 2004) was analysed and IPF patients who had undergone right heart catheterisation (RHC) were identified. $\mathrm{PH}$ was defined as a mean pulmonary arterial pressure $\left(\bar{P}_{\mathrm{pa}}\right) \geqslant 25 \mathrm{mmHg}$ and severe $\mathrm{PH}$ as a $\bar{P}$ pa $>40 \mathrm{mmHg}$. Independent factors associated with $\mathrm{PH}$ were determined.

Of the 3,457 persons listed, 2,525 (73.0\%) had undergone RHC. PH affected $46.1 \%$ of subjects; $\sim 9 \%$ had severe $\mathrm{PH}$. Variables independently associated with mild-to-moderate $\mathrm{PH}$ were as follows: need for oxygen, pulmonary capillary wedge pressure $(\underline{P c w})$ and forced expiratory volume in one second $\left(F E V_{1}\right)$. Independent factors related to severe $\mathrm{PH}$ included the following: carbon dioxide tension, age, $\mathrm{FEV}_{1}, \underline{P}_{\mathrm{pcw}}$, need for oxygen and ethnicity. A sensitivity analysis in subjects with $\underline{P}_{p c w}<15 \mathbf{m m H g}$ did not appreciably alter the present findings.

Pulmonary hypertension is common in idiopathic pulmonary fibrosis patients awaiting lung transplant, but the elevations in mean pulmonary arterial pressure are moderate. Lung volumes alone do not explain the pulmonary hypertension. Given the prevalence of pulmonary hypertension and its relationship with surrogate markers for quality of life (e.g. activities of daily living), future trials of therapies for this may be warranted.

KEYWORDS: Epidemiology, haemodynamics, idiopathic pulmonary fibrosis, pulmonary hypertension

I diopathic pulmonary fibrosis (IPF) is a disease of unknown aetiology associated with progressive parenchymal fibrosis [1]. Patients with IPF face substantial morbidity and mortality, and report substantially impaired quality of life [2]. Lung transplant (LT) represents the lone intervention that potentially improves survival in IPF [3].

Pulmonary hypertension (PH) is evolving as an important factor that can adversely affect outcomes in chronic lung disease. In advanced chronic obstructive pulmonary disease (COPD), therapy directed at controlling the pulmonary artery pressure has been recommended [4]. In fibrotic lung diseases more akin to IPF, such as sarcoidosis, secondary $\mathrm{PH}$ is common and is a marker for early death $[5,6]$.

Less is known about $\mathrm{PH}$ in IPF. Epidemiologically, several retrospective analyses indicate that PH in IPF may be frequent [7-9]. Illustrating the emerging interest in PH and IPF, GHOFRANI et al. [10] examined the impact of sildenafil on pulmonary haemodynamics in lung fibrosis and concluded that it caused pulmonary vasodilation and improved gas exchange. Others have also explored inhaled agents in IPF related to $\mathrm{PH}$ [11].

With the advent of newer options for treating $\mathrm{PH}$, coupled with the lack of effective therapies for $\mathrm{IPF}$, targeting $\mathrm{PH}$ appears attractive. However, before studying interventions it is important to define the prevalence and extent of this process. With improved information regarding the prevalence of and clinical factors associated with $\mathrm{PH}$ in IPF, clinicians can better determine whom to evaluate for $\mathrm{PH}$, and researchers can design more frequency of $\mathrm{PH}$ in IPF, the present authors retrospectively analysed the United States LT registry. The specific objectives were to describe the prevalence of $\mathrm{PH}$ in IPF, to assess the severity of $\mathrm{PH}$ in this population and to identify clinical variables that correlated with $\mathrm{PH}$.

\section{METHODS}

\section{Subjects}

Patients with PH and IPF were identified by reviewing the LT registry maintained by the appropriate clinical trials. Thus, to explore the
AFFILIATIONS

*Pulmonary and Critical Care Medicine Section, Washington Hospital Center,

"Dept of Pulmonary and Critical Care Medicine, Walter Reed Army Medical Center, Washington, DC,

\#United Network for Organ Sharing, Richmond, and

${ }^{+}$INOVA Transplant Center, Fairfax Inova Hospital, Falls Church, VA, USA.

CORRESPONDENCE

A.F. Shorr

Pulmonary and Critical Care

Medicine Section

Washington Hospital Center

Room 2A-38D

110 Irving St, NW

Washington DC 20010

USA

Fax: 12022910386

E-mail: afshorr@dnamail.com

Received:

August 162006

Accepted after revision:

June 252007

STATEMENT OF INTEREST

None declared. 
United Network for Organ Sharing (UNOS) and the Organ Procurement and Transplant Network (OPTN). All adult subjects listed for LT for IPF between January 1995 and June 2004 were included. The diagnoses of IPF were based on the reports of the referring transplant centre. Surgical lung biopsy was not required for the diagnosis of IPF and, given the time period covered, many patients were listed before the development of the recent consensus statement regarding the clinical diagnosis of IPF. The type of proposed LT did not affect eligibility for enrolment in the present study's cohort. Since the focus of the study was $\mathrm{PH}$, patients were required to have undergone right heart catheterisation (RHC) as part of their transplant evaluation. Subjects who did not have an RHC were excluded. There were no additional exclusion criteria.

\section{End-points}

The presence of $\mathrm{PH}$ represented the primary end-point, while severe $\mathrm{PH}$ served as a secondary end-point. $\mathrm{PH}$ was defined as a mean pulmonary artery pressure $\left(\bar{P}_{\mathrm{pa}}\right) \geqslant 25 \mathrm{mmHg}$ and severe $\mathrm{PH}$ as a $\bar{P}_{\mathrm{pa}}>40 \mathrm{mmHg}$.

\section{Study co-variates}

Information concerning demographics and pulmonary function testing was recorded. The forced expiratory volume in one second (FEV1) and the forced vital capacity (FVC) comprised the pulmonary function measures of interest. UNOS does not maintain data regarding the diffusion capacity of the lung for carbon monoxide $(\mathrm{DL}, \mathrm{CO})$ and did not begin collecting the FEV1/FVC ratio until 1999; therefore, it was impossible to include these variables. The partial pressure of carbon dioxide $\left(\mathrm{PCO}_{2}\right)$ and the need for oxygen were also noted. For functional status, the patient's ability to walk $>45 \mathrm{~m}$ in $6 \mathrm{~min}$ and the degree of assistance they required with activities of daily living (ADLs) were gauged. Although an exact distance walked would be preferred for the 6-min walk test, UNOS only records whether the patient had the ability to walk for $45 \mathrm{~m}$. For comorbid diseases, the presence of hypertension, COPD and diabetes were focused on. COPD was defined based on the reports of referring centres and not based on UNOS-calculated FEV1/FVC ratios. Information regarding the pulmonary capillary wedge pressure $(\underline{P} \mathrm{pcw})$ and the cardiac index were also collected. For each of the variables, the model inputs reflect information available at time of listing.

\section{Sensitivity analysis}

Due to the potential for occult left ventricular heart disease and in an effort to control for possible elevations in the $\underline{P}$ pcw directly contributing to elevations in the $\bar{P}_{\mathrm{pa}}$, a sensitivity analysis was conducted in the subgroup of patients with $\underline{P} \mathrm{pcw}$ $<15 \mathrm{mmHg}$. In other words, elevations of the $\bar{P}_{\text {pa }}$ in this subgroup more properly represent isolated $\mathrm{PH}$.

\section{Statistics}

Univariate analyses were performed initially. A Chi-squared test was employed to compare categorical variables. Continuous variables followed a non-normal distribution and, hence, the Wilcoxon test was utilised for comparisons. All tests were two-tailed, and a p-value $<0.05$ was assumed to represent statistical significance.

All factors that were significant were entered in the univariate analysis at the $\leqslant 0.10$ level into the model. A backwards stepwise approach was employed. Candidate variables were removed in a stepwise fashion if $p>0.10$. Co-linearity was assessed via correlation matrices. The model's goodness of fit was assessed with the Hosmer-Lemeshow statistic. The authors also reported 95\% confidence intervals (CIs) around odds ratios (ORs).

\section{RESULTS}

During the 9.5-yr study period, 3,457 persons were listed for LT for IPF. The final cohort comprised 2,525 (73.0\%) subjects who had RHC data available. Those who had undergone RHC were not systematically different from those who did not have an RHC in terms of either demographic characteristics, measures of pulmonary physiology, or assessments of comorbidities. The mean age of the entire study population undergoing RHC was $53.4 \pm 8.7$ yrs and $61.4 \%$ were male. Those not undergoing RHC had a mean age of $54.8 \pm 6.3$ yrs and $63.3 \%$ were male. The mean FVC in both the RHC and non-RHC populations was $\sim 50 \%$. Diabetes was reported to occur in nearly $10 \%$ of all of these patients, irrespective of whether they had an RHC performed. PH was common, affecting $46.1 \%$ of all IPF patients listed for LT. Among those with PH, the $\bar{P}_{\text {pa }}$ measured $34.2 \pm 9.9 \mathrm{mmHg}$. Nearly one in 10 subjects had severe $\mathrm{PH}$.

\section{Nonsevere $\mathbf{P H}$}

Comparisons between those without $\mathrm{PH}$ and those with nonsevere $\mathrm{PH}$ are highlighted in table 1 . Contrasting those without $\mathrm{PH}$ to persons with mild and moderate $\mathrm{PH}\left(\bar{P}_{\mathrm{pa}}\right.$ 25-40 $\mathrm{mmHg}$ ) revealed these groups to be comparable with respect to distribution of sex, while those with mild $\mathrm{PH}$ elevations were slightly younger. $\mathrm{PH}$ was more frequent among African-Americans. Subjects with $\mathrm{PH}$ required more assistance with their ADLs. COPD was 1.31 times (95\% CI 1.03-1.67) more prevalent in the PH cohort. Hypertension also occurred more often in persons with $\mathrm{PH}$, while there was a trend towards an increased frequency of diabetes in those with $\mathrm{PH}$. Corticosteroid use was common in all subjects.

The $\mathrm{PCO}_{2}$ was marginally higher, while the $\mathrm{FVC}$ was slightly lower in PH subjects. However, the FEV1 was lower in persons with $\mathrm{PH}(50.0 \pm 16.5$ versus $52.7 \pm 16.5 \%$ predicted, $\mathrm{p}<0.0001)$. Similarly, individuals with $\mathrm{PH}$ required more supplemental oxygen $\left(2.3 \pm 1.9\right.$ versus $\left.2.9 \pm 2.1 \mathrm{~L} \cdot \mathrm{min}^{-1}, \mathrm{p}<0.0001\right)$. Furthermore, those with $\mathrm{PH}$ in IPF were nearly $80 \%$ more likely (OR $1.78,95 \%$ CI $1.50-2.11, \mathrm{p}<0.0001)$ to require $>3 \mathrm{~L} \cdot \mathrm{min}^{-1}$ of oxygen. Results of invasive haemodynamic monitoring indicated that the cardiac index was equivalent between those with and without $\mathrm{PH}$, although the $\underline{P} \mathrm{pcw}$ was higher in the $\mathrm{PH}$ cohort.

In multivariate analysis (table 2), several variables were independently associated with the presence of $\mathrm{PH}$. The need for supplemental oxygen, the $\underline{P} \mathrm{pcw}$ and the FEV1 correlated with $\bar{P}_{\text {pa. }}$. Those requiring oxygen were significantly more likely to have concomitant PH (OR 1.22, 95\% CI 1.14-1.30, $\mathrm{p}<0.001)$. Similarly, a higher $\underline{P} \mathrm{pcw}$ increased the probability that $\mathrm{PH}$ would be present. Neither the FVC nor the cardiac index showed a relationship with $\mathrm{PH}$. 
TABLE 1 Correlates of pulmonary hypertension

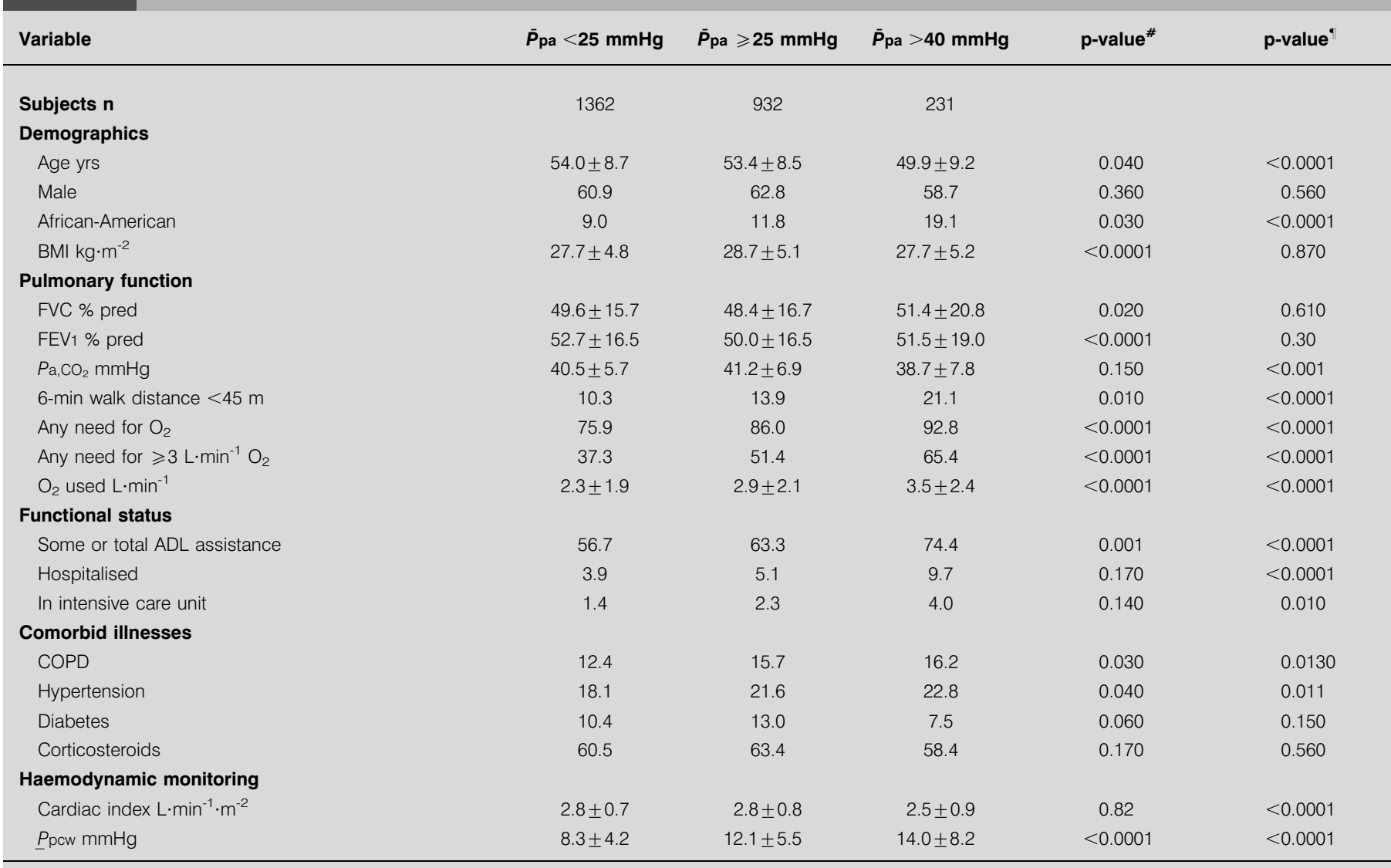

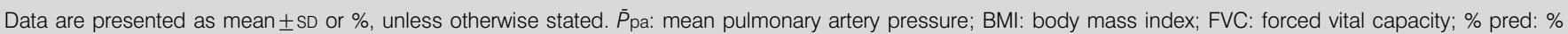
predicted; FEV1: forced expiratory volume in one second; $\mathrm{Pa}_{2} \mathrm{CO}_{2}$ : carbon dioxide arterial tension; $\mathrm{ADL}$ : activities of daily living; COPD: chronic obstructive pulmonary disease; Ppcw: pulmonary capillary wedge pressure. ${ }^{*}: \bar{P}_{\mathrm{pa}}>25$ versus $\leqslant 25 \mathrm{mmHg} ;{ }^{\bullet}: \bar{P}_{\mathrm{pa}}>40$ versus $\leqslant 25 \mathrm{mmHg} . \mathrm{n}=2,525.1 \mathrm{kPa}=0.133 \mathrm{mmHg}$

\section{Severe $\mathbf{P H}$}

Comparisons between those with normal $\bar{P}_{\text {pa }}$ values and patients with severe $\mathrm{PH}$ are also shown in table 1. Many of the differences seen in the analysis with the nonsevere $\mathrm{PH}$ subjects were noted in the comparison between those lacking $\mathrm{PH}$ and those with severe PH. For example, the need for both assistance with ADLs or admission to the hospital at time of listing was more common in severe PH. Hypertension was more often noted in patients with severe $\mathrm{PH}$. It was concerning that ethnicity again correlated with $\mathrm{PH}$. Nearly $20 \%$ of the severe $\mathrm{PH}$ group were African-American versus only $9 \%$ of the
non-PH population (OR 2.37, 95\% CI 1.63-3.46, $\mathrm{p}<0.001)$. In terms of physiology, FVC failed to segregate patients with severe PH from those with normal $\bar{P}$ pa values and was, in fact, numerically higher in those with worse $\bar{P}_{\text {pa }}$ values. The FEV1 did not differ as a function of the $\bar{P}_{\mathrm{pa}}$. Interestingly, although the $\mathrm{PCO}_{2}$ was higher in those with mild and moderate elevations of $\bar{P}$ pa compared with those with normal $\bar{P}_{\text {pa values, }}$ the $\mathrm{PCO}_{2}$ was lowest in the severe $\mathrm{PH}$ patients.

Multivariate analysis (table 3) identified several variables independently linked with severe $\mathrm{PH}$. Unlike the present

TABLE 2 Independent correlates of mild-to-moderate pulmonary hypertension\#

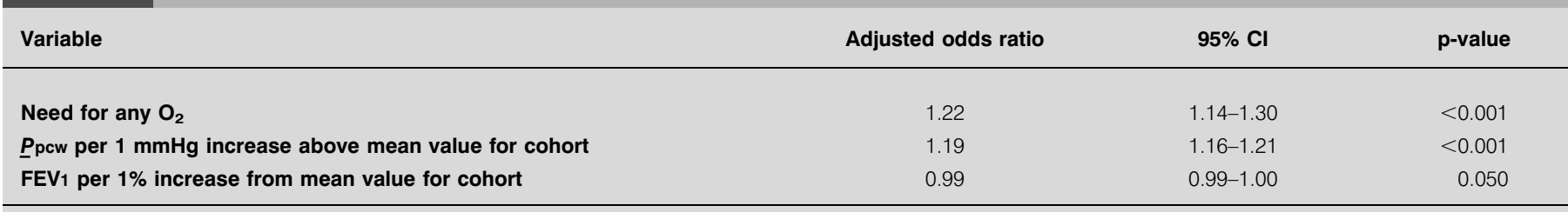

$\mathrm{Cl}$ : confidence interval; $P$ pcw: pulmonary capillary wedge pressure; FEV1: forced expiratory volume in one second. The Hosmer-Lemeshow Goodness-of-Fit test yields a Chi-squared value of $14.8(p=0.06) .{ }^{*}$ : defined as mean pulmonary artery pressure between $25-40 \mathrm{mmHg} . \mathrm{n}=2,296.1 \mathrm{kPa}=0.133 \mathrm{mmHg}$. 
observations in the nonsevere $\mathrm{PH}$ cohort, it was noted that ethnicity significantly correlated with the presence of severe $\mathrm{PH}$. Specifically, African-Americans were twice as likely to have severe $\mathrm{PH}$ compared with Caucasians (OR 1.93, 95\% CI 1.02-3.66, $p=0.043)$. The need for supplemental oxygen also identified subjects who were more likely to have severe elevations in $\bar{P}_{\text {pa. }}$. With respect to invasive haemodynamics, $\underline{P}$ pcw remained a significant factor associated with a diagnosis of severe $\mathrm{PH}$. While cardiac performance did not appear to be related to severe $\mathrm{PH}$, age, $\mathrm{PCO}_{2}$ and FEV1 were independently associated with the presence of severe $\mathrm{PH}$.

\section{Sensitivity analysis}

Out of the entire population, $\sim 18 \%$ had a $\underline{P}$ pcw $>15$. After excluding patients with $\underline{P}_{\mathrm{pcw}}>15$, the results of the logistic regressions to identify factors independently related to $\mathrm{PH}$ varied somewhat from those observed in the entire $\mathrm{PH}$ population. Table 4 indicates variables associated with mildto-moderate $\mathrm{PH}$ not associated with an increased $\underline{P}$ pcw. As seen in the overall analysis of $\mathrm{PH}$, oxygen use, FEV1 and $\underline{P} \mathrm{pcw}$ remained significantly correlated with isolated $\mathrm{PH}$. Additionally, the need for assistance with ADLs and the presence of hypertension now appeared linked to mild-tomoderate $\mathrm{PH}$. Table 5 reveals the findings from the multivariate analysis in persons with severe, isolated $\bar{P}_{\text {pa elevations. }}$ Younger age along with the $\mathrm{PCO}_{2}$ were "protective". In general, factors independently associated with severe $\mathrm{PH}$ also correlated with severe, isolated PH. For example, AfricanAmericans again faced a nearly two-fold increased probability of having severe $\mathrm{PH}$. In addition to variables noted in the overall analysis of severe $\mathrm{PH}$, a low 6-min walk distance and need for assistance with ADLs additionally were associated with the presence of severe, isolated $\mathrm{PH}$.

\section{DISCUSSION}

This large retrospective analysis reveals that $\mathrm{PH}$ is common in persons with IPF awaiting LT. Although PH affects $\sim 45 \%$ of these subjects, severe $\mathrm{PH}$ is relatively infrequent. Multivariate analysis indicates that several variables are independently associated with $\mathrm{PH}$ and severe $\mathrm{PH}$, but that only three factors, FEV1, the need for supplemental oxygen and an elevated $\underline{P} \mathrm{pcw}$, consistently correlate with the presence of $\mathrm{PH}$ across varying degrees of severity. In the sensitivity analysis of persons who meet the definition for isolated $\mathrm{PH}$ (e.g. elevated $\bar{P}_{\mathrm{pa}}$ and $\underline{P} \mathrm{pcw}$ $<15 \mathrm{mmHg}$ ), FEV1, need for assistance with ADLs, $\underline{P}_{\mathrm{pcw}}$ and oxygen use appear linked to all degrees of this.

Several earlier analyses have gauged the prevalence of $\mathrm{PH}$ in IPF. KING et al. [7] found that $20 \%$ of those with IPF had evidence of $\mathrm{PH}$. However, the presence of $\mathrm{PH}$ was only assessed by chest radiography [7]. In a study of 25 persons, AgARWAL et al. [12] determined that $36 \%$ had $\mathrm{PH}$; however, these authors [12] relied only on echocardiography, which may overestimate or underestimate pulmonary arterial pressure in persons with interstitial lung disease [13]. In a larger analysis also relying on echocardiography, NADROUs et al. [8] concluded that $\mathrm{PH}$ was common in advanced IPF and that it correlated with both a lower DL,CO and a lower resting arterial oxygen tension [8]. The study by NADROUs et al. [8] was limited in that there was the potential for substantial selection bias as only 136 $(28 \%)$ out of 487 of patients with IPF had undergone echocardiography [8]. Finally, relying on RHC, LETTIERI et al. [9] noted that PH was documented in approximately one-third of persons with IPF [9]. Additionally, these authors demonstrated that even moderate elevations of $\bar{P}_{\text {pa }}(>25 \mathrm{mmHg})$ correlated with increased mortality [9].

The present analysis confirms the findings of these earlier reports and clearly demonstrates that $\mathrm{PH}$ is a common complication of IPF. More importantly, the present study builds on these efforts, in that it represents the largest experience with RHC in IPF. As a result, the larger sample size used herein allows more certainty as to the estimation of the prevalence of $\mathrm{PH}$ in IPF. Additionally, since only RHC was

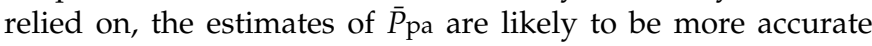
than those based solely on echocardiography. Moreover, all prior studies represented the experience of single centres. Since UNOS and OPTN serve as the registry for all USA LTs, it was possible to draw on patients seen at multiple centres.

Pathophysiologically, the factors independently related to the presence of $\mathrm{PH}$ suggest some mechanisms as to the evolution of $\mathrm{PH}$ in IPF. The correlation between lower FEV1 and $\mathrm{PH}$ implies that superimposed airflow limitation may accelerate potential elevations in the $\bar{P}$ pa. Furthermore, an abnormal $\mathrm{PCO}_{2}$ may mean that underlying gas exchange abnormalities increase the risk for $\mathrm{PH}$. However, for both of these factors the statistical relationships observed may not be clinically

\section{TABLE 3 Independent correlates of severe pulmonary hypertension ${ }^{\#}$}

\begin{tabular}{|c|c|c|c|}
\hline Variable & Adjusted odds ratio & $95 \% \mathrm{Cl}$ & p-value \\
\hline $\mathrm{PCO}_{2}$ per 1-point increase & 0.92 & $0.89-0.96$ & 0.0001 \\
\hline Age at listing & 0.95 & $0.92-0.97$ & $<0.0001$ \\
\hline FEV 1 per $1 \%$ increase from mean value for cohort & 0.96 & $0.93-0.99$ & 0.003 \\
\hline Ppcw per $1 \mathrm{mmHg}$ increase above mean value for cohort & 1.22 & $1.17-1.28$ & $<0.001$ \\
\hline Any $\mathrm{O}_{2}$ needed & 1.29 & $1.12-1.49$ & 0.004 \\
\hline $\mathrm{O}_{2}$ need $>3 \mathrm{~L} \cdot \mathrm{min}^{-1}$ & 1.89 & $1.03-3.47$ & 0.041 \\
\hline African-American & 1.93 & $1.02-3.66$ & 0.043 \\
\hline
\end{tabular}




\section{TABLE 4 Independent correlates of mild-to-moderate isolated pulmonary hypertension}

\begin{tabular}{|c|c|c|c|}
\hline Variable & Adjusted odds ratio & $95 \% \mathrm{Cl}$ & p-value \\
\hline FEV 1 per $1 \%$ increase from mean value for cohort & 0.99 & $0.990-0.997$ & 0.006 \\
\hline Need for assistance with ADLs & 1.24 & $1.00-1.54$ & 0.047 \\
\hline Hypertension & 1.33 & $1.03-1.72$ & 0.030 \\
\hline $\mathrm{O}_{2}$ need $>3 \mathrm{~L} \cdot \mathrm{min}^{-1}$ & 2.84 & $2.30-3.50$ & $<0.001$ \\
\hline
\end{tabular}

meaningful; differences in the mean values for these variables

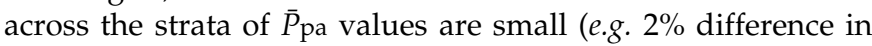
FEV1) and thus any correlation is likely to be speculative rather than causative. It therefore appears likely that, in IPF, additional causes for elevated $\bar{P}$ pa values exist. Other investigators have reached similar conclusions when exploring the mechanisms for $\mathrm{PH}$ in COPD. CHAOUAT et al. [14] performed extensive evaluations in persons with severe COPD and $\mathrm{PH}$. Their data demonstrated that factors other than the extent of the COPD itself contributed to $\bar{P}_{\text {pa }}$ increases [14]. With respect to COPD, patients with concurrent IPF and emphysema may be at particularly increased risk for PH. For example, COTTIN et al. [15] noted that nearly half of such subjects had $\mathrm{PH}$. Although no such independent association between COPD and $\mathrm{PH}$ was found in the present study, the link between FEV1 and $\mathrm{PH}$ indicates that patients with both conditions may be at high risk for $\mathrm{PH}$.

The following three factors correlated with $\mathrm{PH}$ across the range of pressure elevations: FEV1, the need for supplemental oxygen and $\underline{P}$ pcw. In the cohort without elevated $\underline{P}$ pcw, assistance with ADLs also appeared important. The interaction between ADLs and $\mathrm{PH}$ is likely to underscore the morbidity burden associated with $\mathrm{PH}$. In other words, inability to perform ADLs does not cause $\mathrm{PH}$ but reflects the burden of this on a patient's performance status [2].

Multiple potential factors could account for the association between the need for supplemental oxygen and PH. This may indicate the impact of chronic pulmonary vasoconstriction due to alveolar hypoxia, although the connection between chronic alveolar hypoxia and pulmonary vasoconstriction has not been conclusively demonstrated. It could also represent that, due to regulations regarding the use of supplemental oxygen, these patients might simply be more likely to have oxygen prescribed as a result of underlying cor pulmonale. Conversely, PH may cause hypoxaemia due to both a low cardiac output and low central venous oxygen saturations, along with increased perfusion of pulmonary shunt vessels or low ventilation/perfusion ratio areas.

The interaction between $\underline{P}$ pcw and $\mathrm{PH}$ appears to be more complicated. The correlation between the two suggests that some component of left ventricular (LV) dysfunction is contributing to $\mathrm{PH}$ in IPF. However, several aspects of the present data reveal that more nuanced issues may be involved.
First, there was no independent relationship between $\mathrm{CI}$ and $\mathrm{PH}$. If LV dysfunction was contributing to $\mathrm{PH}$, one would predict that the CI would be lower in those with $\mathrm{PH}$. Secondly, despite the association between $\mathrm{PH}$ and $\underline{P} \mathrm{pcw}$, the average $\underline{P}$ pcws in the population were, nonetheless, in the normal range. Thirdly, the gradient between the $\bar{P}_{\text {pa }}$ and the average $\underline{P}$ pcw in this population was higher than one would expect if this process represented passive congestion. This discordance underscores that some process other than purely LV dysfunction must be contributing to the PH. Similarly, since the population analysed is relatively selected as these subjects are felt to be candidates for LT, overt congestive heart failure is unlikely to be present. The sensitivity analysis of patients meeting criteria for isolated $\mathrm{PH}$ used in the present study shows a persistent relationship between $\bar{P}_{\text {pa }}$ and $\underline{P}$ pcw. Given that, by design, these persons have no elevations of the $\underline{P} \mathrm{pcw}$,

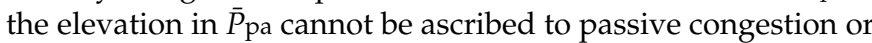
occult LV dysfunction.

It might be hypothesised that progressive parenchymal destruction would lead to PH in IPF. However, in the present study, a strong correlation between FVC and $\mathrm{PH}$ was not observed. Similarly, the two other studies expressly exploring the nexus between FVC and PH, those by NADrOus et al. [8] and by LETTIERI et al. [9], also did not report a positive relationship between FVC and $\mathrm{PH}$. Likewise, in an analysis of the physiology of $\mathrm{PH}$ in lung fibrosis, LEUCHTE et al. [16] did not note a correlation between $\mathrm{PH}$ and FVC. These results, together with data from the present study, imply that issues other than decrements in FVC contribute to PH. Alternatively, the failure of the present study to detect an interaction between FVC and PH may reflect that many subjects already had advanced IPF with low FVC. However, this is doubtful, since both the mean FVC and SD around the FVCs across all three populations studied (normal, mild-to-moderate $\mathrm{PH}$ and severe $\mathrm{PH})$ were similar.

African-Americans faced a greater probability of suffering from severe $\mathrm{PH}$. This observation is troubling, given that earlier analyses noted previously show that $\mathrm{PH}$ portends worse outcomes in IPF. This connection between severe $\bar{P}$ pa elevations and ethnicity might reflect later referral during the disease's natural history. However, that ethnicity independently remained strongly associated with severe $\mathrm{PH}$ (irrespective of the $\underline{P}$ pcw) after controlling for other conventional markers of severity of illness and performance status in IPF 
TABLE 5 Independent correlates of severe isolated pulmonary hypertension

\begin{tabular}{|c|c|c|c|}
\hline Variable & Adjusted odds ratio & $95 \% \mathrm{Cl}$ & p-value \\
\hline $\mathrm{PCO}_{2}$ & 0.88 & $0.85-0.92$ & $<0.001$ \\
\hline Ppcw per $1 \mathrm{mmHg}$ increase above mean value for cohort & 1.26 & $1.18-1.34$ & $<0.001$ \\
\hline 6-min walk distance $<45 \mathrm{~m}$ & 1.71 & $1.00-2.94$ & 0.050 \\
\hline Need for assistance with ADLs & 1.78 & $1.15-2.74$ & 0.010 \\
\hline
\end{tabular}

$\mathrm{Cl}$ : confidence interval; $\mathrm{PCO}_{2}$ : carbon dioxide tension; $\underline{P}$ pcw: pulmonary capillary wedge pressure; ADLs: activities of daily living. The Hosmer-Lemeshow Goodness-of-Fit test yields a Chi-squared value of $5.9(p=0.66) . n=1,308.1 \mathrm{kPa}=0.133 \mathrm{mmHg}$.

argues against this conclusion. Differences in access to care may in some way also contribute to this finding, and this suggests that clinicians must work to ensure that there is not some form of subtle discrimination in their management of African-Americans suffering from IPF. Alternatively, differences in vascular biology could help to explain this finding. African-Americans, for instance, have been reported to respond differentially, when compared with Caucasians, to certain vasodilators administered in the treatment of heart failure [17]. Future investigations are necessary to explore the meaning and cause of this observation.

The present study has several limitations. The retrospective design exposes the analysis to bias. However, unlike the data generated in other reports dealing with $\mathrm{PH}$ in IPF, the UNOS/ OPTN registry represents data collected contemporaneously at listing. Thus, recall bias and coding bias are unlikely. Additionally, some of the patients listed for LT for IPF may not have had IPF, but some other form of interstitial lung disease. However, the demographic distribution indicates that this is not likely, as the cohort resembles the general composition of patients with IPF. Information was also lacking on certain variables that could have been of interest, such as DL,CO. Consequently, the noting of a relationship between the need for supplemental oxygen and $\mathrm{PH}$ may simply indicate that the need for supplemental oxygen is a surrogate for a lower DL,CO. Finally, only patients who were actually listed for LT were studied. Information on potential subjects not evaluated for LT was not available; this limits the generalisability of the present conclusions. If the cohort included persons with less advanced IPF, it might have been possible to detect a correlation between lung function and PH. Despite these considerations, the present study is the largest experience with RHC in IPF.

In conclusion, pulmonary hypertension is common in idiopathic pulmonary fibrosis. Generally, the degree of pulmonary hypertension in idiopathic pulmonary fibrosis is mild to moderate, with few subjects developing severe pulmonary hypertension by the time of listing for lung transplant. Several clinical variables correlate with the presence of pulmonary hypertension. In light of the prevalence of pulmonary hypertension in idiopathic pulmonary fibrosis, coupled with the absence of highly effective therapies for this disease, clinical trials of agents directed at controlling the mean pulmonary arterial pressure in idiopathic pulmonary fibrosis seem warranted.

\section{REFERENCES}

1 American Thoracic Society. Idiopathic pulmonary fibrosis: diagnosis and treatment. International consensus statement. American Thoracic Society (ATS), and the European Respiratory Society (ERS). Am J Respir Crit Care Med 2000; 161: 646-664.

2 Swigris JJ, Gould MK, Wilson SR. Health-related quality of life among patients with idiopathic pulmonary fibrosis. Chest 2005; 127: 284-294.

3 Hosenpud JD, Bennett LE, Keck BM, Edwards EB, Novick RJ. Effect of diagnosis on survival benefit of lung transplantation for end-stage lung disease. Lancet 1998; 351: 24-27.

4 Higenbottam T. Pulmonary hypertension and chronic obstructive pulmonary disease: a case for treatment. Proc Am Thorac Soc 2005; 2: 12-19.

5 Shorr AF, Davies DB, Nathan SD. Predicting mortality in patients with sarcoidosis awaiting lung transplantation. Chest 2003; 124: 922-928.

6 Shorr AF, Helman DL, Davies DB, Nathan SD. Pulmonary hypertension in advanced sarcoidosis: epidemiology and clinical characteristics. Eur Respir J 2005; 25: 783-788.

7 King TE Jr, Tooze JA, Schwarz MI, Brown KR, Cherniack RM. Predicting survival in idiopathic pulmonary fibrosis: scoring system and survival model. Am J Respir Crit Care Med 2001; 164: 1171-1181.

8 Nadrous HF, Pellikka PA, Krowka MJ, et al. The impact of pulmonary hypertension on survival in patients with idiopathic pulmonary fibrosis. Chest 2005; 128: Suppl. 6, 616S-617S.

9 Lettieri CJ, Nathan SD, Barnett SD, Ahmad S, Shorr AF. Prevalence and outcomes of pulmonary arterial hypertension in advanced idiopathic pulmonary fibrosis. Chest 2006; 129: 746-752.

10 Ghofrani HA, Wiedemann R, Rose F, et al. Sildenafil for treatment of lung fibrosis and pulmonary hypertension: a randomised controlled trial. Lancet 2002; 360: 895-900.

11 Olschewski H, Ghofrani HA, Walmrath D, et al. Inhaled prostacyclin and iloprost in severe pulmonary hypertension secondary to lung fibrosis. Am J Respir Crit Care Med 1999; 160: 600-607. 
12 Agarwal R, Gupta D, Verma JS, Agarwal RW, Jindal SK. Noninvasive estimation of clinically asymptomatic pulmonary hypertension in idiopathic pulmonary fibrosis. Indian J Chest Dis Allied Sci 2005; 47: 267-271.

13 Arcasoy SM, Christie JD, Ferrari VA, et al. Echocardiographic assessmentof pulmonaryhypertensionin patients withadvanced lung disease. Am J Respir Crit Care Med 2003; 167: 735-740.

14 Chaouat A, Bugnet AS, Kadaoui N, et al. Severe pulmonary hypertension and chronic obstructive pulmonary disease. Am J Respir Crit Care Med 2005; 172: 189-194.
15 Cottin V, Nunes H, Brillet PY, et al. Combined pulmonary fibrosis and emphysema: a distinct underrecognised entity. Eur Respir J 2005; 26: 586-593.

16 Leuchte $\mathrm{HH}$, Neurohr C, Baumgartner R, et al. Brain natriuretic peptide and exercise capacity in lung fibrosis and pulmonary hypertension. Am J Respir Crit Care Med 2004; 170: 360-365.

17 Taylor AL, Ziesche S, Yancy C, et al. Combination of isosorbide dinitrate and hydralazine in blacks with heart failure. N Engl J Med 2004; 351: 2049-2057. 